\title{
A multimodal transportation system routing implemented in waste collection
}

\author{
Masoud Rabbani*, Shadi Sadri and Hamed Rafiei
}

School of Industrial and Systems Engineering, College of Engineering, University of Tehran, Iran

\begin{tabular}{l}
\hline C H R O N I C L E \\
\hline Article history: \\
Received June 25, 2015 \\
Received in revised format: \\
June 28, 2015 \\
Accepted August 20, 2015 \\
Available online \\
August 25 2015 \\
\hline Keywords: \\
Vehicle routing problem \\
Waste management \\
Multimodal transportation system \\
Genetic algorithm \\
Tabu search \\
\end{tabular}

\section{A B S T R A C T}

\begin{abstract}
Waste collection is an important municipal service that charges large expenditures to waste management (WM) system. In this study, a hierarchical structure is proposed in order to minimize total cost of waste collection routing problem. Moreover, in second stage destructive environmental effects of waste transportation are minimized concurrently through taking advantage of a road/rail transportation system. In the proposed multimodal transportation system, waste packs are transferred to final destination while travel time and risk of environmental threatening is minimized. The discussed problem is formulated mathematically in two stages. In the first stage, a household waste collection routing problem is formulated while, in second stage a multimodal transportation system is routed to transfer waste packs to final destination through roads and railroads. In order to solve the proposed NP hard models, an improved genetic algorithm is developed. Comparison of the obtained results with those of GAMS for small-size samples validates the proposed models.
\end{abstract}

(c) 2016 Growing Science Ltd. All rights reserved.

\section{Introduction}

In a supply chain, transportation system connects different components of any system. One of the major challenges in designing a transportation system for a supply chain is selection of vehicle types and routing. For the first time, Dantzig and Ramser (1959) discussed this issue as the Vehicle Routing Problem (VRP), and over the years researchers have developed several variations of the problem. In some researches, authors have developed a new field in routing problems by combining waste management and vehicle routing problems. In such studies, the attempt is to route the waste collection vehicles. Wastes are derived from several manufacturing, industrial, service, residential and healthcare activities (Maimoun et al., 2013) which can be classified into three groups generally: Residential, Commercial, and Industrial (Sahoo et al., 2005). Residential wastes are derived from daily household activities and require a daily collection plan. On the other hand, commercial waste collection involves servicing customers as markets, office buildings and hospitals. Industrial wastes are mainly produced in factories, industrial and production sites. The notable difference of industrial wastes from other types

\footnotetext{
* Corresponding author.

E-mail address: mrabani@ut.ac.ir (M. Rabbani) 
is the size of bins used to collect them. According to the bin size, each container has capacity for no more than one customer and it necessitates a special collection plan.

By considering the inevitability of waste production in everyday life and their destructive effect on the environment, along with other threats to people health, associate governors and executive managers are required to route vehicles optimally in order to reduce 1) required time for waste collection operations, and 2) traffic. Traffic regulations is one of the limitations that impede vehicle routing for Residential and Commercial waste transportation in the city. One-way streets and prohibited turns are examples of such restrictions. In addition, vehicles cannot use some routes due to vehicle weight and width of the passageway (Sniezek et al., 2006). Such limitations are also passed for intercity routes which affect transportation activities. Hence, it is necessary to use another type of fleet to transit along restricted paths. Multimodal transportation approach is a good solution for maximizing accessibility and reduction of travel distance. Multimodal transportation is defined as transporting products by two or more modes of transport systems from depot to customers (Lowe, 2005). Applying a multimodal transportation system leads to take advantage of different transit systems.

Developing multimodal transportation system to convey wastes is an approach to increase access to different sites and reduce destructive effects of an invariable transportation system. Implementing a multimodal transportation system enables transporting wastes through different types of routes by reduction of undesirable impacts on environment and health. Optimal selection and scheduling of independent parts can enhance system performance. In this study, a rail/road transportation system is considered for transferring wastes with objective function of minimizing environment destruction and health threatening. In this regard, two qualitative factors, probability of accident and emission pollution rate, are considered. Here a hierarchical strategy is developed that in the first stage, residential waste collection routing problem is solved for a nonhomogeneous fleet including handcarts, and vehicles in small and medium size. In the second stage, a multimodal transportation system is implemented to convey waste packs from disposal site to final destination. Reusability of some types of solid wastes causes using them as raw materials for some industries. This fact is considered in this study and after separating and packing solid wastes in disposal sites, they are delivered to a manufacturer as final destination. In order to solve the considered problem, an improved genetic algorithm, which is hybridization of genetic algorithm and heuristic algorithms, is developed.

The remainder of study is organized as follow. In section 2 a review of literature is proposed. Section 3 develops a hierarchical structure in which a waste collection routing problem and multimodal transportation system routing problem are formulated mathematically. Implemented solving methods to solve NP hard models are explained in section 4 and results of some numerical examples are reported in section 5. A sensitivity analysis of system cost to each cost component is provided in section 6 in order to validate the proposed models and algorithms. Finally the paper is concluded in section 7.

\section{Background}

Nowadays increasing people's interest to live in municipals, leads to increment of waste which poses their health and environment to a serious threat. Numerous studies on optimization of waste collection routing problems have been accomplished in recent decades. In the following of this section, we reviewed some of these studies. Nema et al. (1999) studied the planning and design of hazardous waste management system considering an illustrative case example. They presented an improved formulation based on multi-objective integer programming approach to optimize the system. Residential waste collection routing problems are usually modeled using arc routing approach. Tung et al. (2000) developed a new model for residential waste collection in Hanoi, Vietnam. They modeled the problem mathematically using operations research techniques and applied a two-phase heuristic method, including a constructive phase followed by an improvement phase. In some studies, there is no information about data. Ghose et al. (2005) proposed a GIS optimal routing model to determine the 
minimum cost/distance efficient collection paths for transporting the solid wastes. Nuortio et al. (2006) studied a waste collection problem with random parameters. The authors employed data from previous records for weight and location of bins to estimate average cumulative wastes in each bin located in a street. They developed a node-based routing model and used a metaheuristic method called guided variable neighborhood thresholding to solve the model. Health-care waste collection is another aspect of waste collection routing problems which was considered by Alagoz et al. (2008) in Istanbul. They implemented special software programs called MapInfo and Roadnet to solve the scheduling and route optimization problem. Tavares et al. (2009) optimized the routing network used for waste collection and transportation in order to minimize the fuel consumption. They applied GIS 3D route modelling software to optimize the problem.

In majority of studies, the objective is to minimize costs, travel distance and time. A portion of the required time in waste collection process is dedicated to loading and unloading activities. Faccio et al. (2011) studied the waste collection problem by considering loading and unloading times aim to minimize these times. They proposed a multi-objective model with dynamic and real data that is updated periodic and developed a heuristic algorithm to solve the model. Over the years none of the researchers has considered a penalty cost for servicing customers out of time windows. Moustafa et al. (2013) discussed this issue for the first time. They defined penalty cost of arriving at customer's node earlier than the specified time as operational cost and tried to reduce waste collection time and fleet size using TransCAD software to calculate optimal solution for the problem.

In addition to common routing-related issues in designing collection routes, Ramos et al. (2014) considered the economic and environmental concerns. They developed a decomposition solution approach to solve the proposed bi-objective model and studied six different scenarios regarding different objective functions. Bing et al. (2014) proposed a tabu search heuristic to redesign the collection routes. In this study, authors compared the collection alternatives by a scenario study approach and validated the proposed approach. In another study, Bing et al. (2014) minimized both transportation cost and environmental impact of waste collection by implementing a scenario-based strategy on a mixed integer linear programming. In proposed study, they considered plastic wastes and their separation process through a reverse logistics network. Besides this, Hemmelmayr et al. (2014) considered bin allocation along with route design. They considered different types of bins and a tradeoff between associated transportation cost and bin allocation cost. They decided about both variables in a hierarchical structure. On the other hand, Hsieh et al. (2014) focused on service frequency and various types of collection namely one-side collection, two-side collection and no collection. In this study, the authors attempt to minimize total completion time of vehicles and workload balance among vehicles based on their capacity. For the discussed problem, an immune based approach was developed to solve the model and design an optimal network.

Because of width and weight limitation for some passageways, in some urban areas, using large size vehicles is impossible. Thus, smaller vehicles must be used to service customers. Sniezek et al. (2006) considered this constraint and classified facilities in terms of size into different groups and proposed an optimal approach called combinatorial approach. Another limitation in routing problems is traffic regulations. Bautista et al. (2008) took these rules into account and solved an arc routing problem in Barcelona, Spain by converting it to a node routing problem. They applied an Ant Colony heuristic to propose optimal routes for urban waste collection by considering mentioned traffic restrictions in a city. Liu et al. (2012) considered access restrictions and tried to reduce travel distances. They proposed a multiple classified ant colony algorithm.

As mentioned before, multimodal transportation system is a good solution to eliminate the accessibility constraints. In some studies, the advantage of implementing a multimodal transportation system is considered. In multimodal transportation systems, it is possible to select routes with minimum travel times in various types. However, managers should be careful about considering the transportation time 
from one type to another. This issue has been focused by Ziliaskopoulos et al. (2000). They developed a multiple optimal routing algorithm that improves time factor to deal with this problem. Arnold et al. (2004) dealt with multimodal transportation system from another perspective. They developed a binary mathematical model by incorporating railway/road terminal location assumption and employed heuristic methods to solve the model. Galvez-Fernandez et al. (2009) seek optimization of multiple transportation services in Europe by applying the Transfer Graph approach for multimodal timedependent transportation networks. Proposed approach is two-phase in which the first phase calculates proper routes in every single-model network and the second phase solves the model for optimality by constructing a simpler single-model graph. In addition, Dijkstra algorithm is suggested as a solution method for multiple transportation problems. Despite the reduction of computation time, requiring a large computational memory limits its capability in solving large-size problems. To eliminate this problem, Ayed et al. (2009) developed Ant Colony optimization method which requires lower memory but longer computation time. Ayed et al. (2011) extended previous research by developing a solution approach based on combination of Dijkstra algorithm and Ant Colony optimization method. Their combinatorial approach balance computation time and required computational memory. Another multiobjective multi-level Taguchi Genetic Algorithm has been developed by Xiong et al. (2012). They utilized Taguchi method to increase speed and stability of the Genetic Algorithm in calculating initial solution. Thus, the proposed approach is capable of producing a set of transportation routes using a reasonable combination of transportation types.

A brief review of conducted studies on vehicle routing problems in waste transportation systems indicates that no researches concentrated on advantages of multimodal transportation systems in this field. Such systems can benefit from different transportation methods simultaneously. In this research, a hierarchical structure is developed by considering a rail/road transportation system, to transfer wastes from production site to transshipment, and then to disposal and recycling sites in order to minimize operational costs, air pollution by vehicles and destructive environmental effects. There are different types of routing problems with a rich literature. (To see more refer to Bodin et al., 1982; Siddiqui et al., 2015; Saleh et al., 2014).

\section{Problem Description}

In this study authors are motivated to apply a multimodal transportation system for the first time in waste collection problem regard to minimize the environmental destructive effects and take advantage of different modes of transit systems. In the proposed study a road/rail transportation system is developed to collect residential wastes and transfer to the final destination. Collection activities are organized in two shifts and a heterogeneous fleet including handcarts and vehicles with small and medium sizes are implemented to serve the households in point-to-point manner. In reality there are some narrow passageways which cannot be travelled by vehicles. In this study, associated managers determine the desired vehicle class to service each node by evaluating the width of path represented by $\alpha_{i}$. We cosider a boundary to determine vehicle class equals to 0.3. Values less than the boundary represent the requirement to handcarts to service the nodes. While for others, all vehicle classes are utilized. In case of capacity shortage, vehicles travel to landfill in order to unload the collected wastes. In real situation, municipalities operate from different depots and implement multiple collection vehicles. Hence studying this variant of VRP is as useful as classic ones.

In the considered problem, at the start of a shift each vehicle is provided with a schedule, based on which vehicle crew start a trip at 8:00 AM from a depot and direct to collect wastes from households. According to the specified width parameter $\left(\alpha_{i}\right)$, nodes are served by desired vehicles. Full-loaded vehicles travel to disposal site and after discarding, start another route. The first trip starts at depot and ends at disposal site, whereas subsequent trips start and end at disposal site. At the end of shift (4:00 PM), unloaded vehicles return to depot and finish their tour. Not serving customers during shift charges penalty cost, proportional to not collected waste volume. Second shift starts at 4:00 PM and vehicles 
serve the dispersed waste bins in city and due to the size of bins, handcarts are not utilized. Collection activities proceeds to 12:00 AM and after discarding wastes in disposal sites, vehicles return to depot from which originate.

Due to the permanence of solid wastes, it is presumed solid wastes are separated and packed in landfills. Being packed, containers convey waste packs to loading rail stations. In containers routing, distance factor and probability of accident in a path affect selecting the traverse nodes. Regards to the importance of environment health and increment of transport speed, loading has been done in stations through which few passengers traverse or in hours that less passengers ply. Assumptions of the proposed structure are as below:

- Multiple depots and disposal sites are considered to cover an extended area;

- Collection activities are divided into 2 shifts. In the first shift household wastes are collected whereas in second one waste bins are emptied;

- Nodes along narrow passageways are served by handcarts while others can be served by all vehicle classes;

- In case of not servicing nodes in related time window a penalty cost will be imposed on the system; and

- Due to the health of environment and passengers, in train routing stations with low traffic are considered;

The considered residential waste collection problem by implementing a multimodal transportation system is formulated in a hierarchical structure. This study is an extension of Salhi et al., (2013) in waste management area. In the first stage, the collection fleet are routed with the objective function of minimizing system cost. The network of problem is represented by a directed graph, $G(V, E, M, T)$, where $V=\left\{I^{D}, I^{h}, I^{v h}, I^{B}, I^{i f}\right\}$ is the set of nodes, $E$ is the set of arcs, $M$ is the set of vehicle classes, and $T$ is the discretized shift. $I^{D}$ represents depots, where $I^{h}, I^{v h}$ are demand nodes served by handcarts and all vehicle classes, respectively. The locations of waste bins are shown as $I^{B}$ and disposal sites are represented by $I^{\text {if }}$. Due to diversification of collection equipment, $M$ includes $M^{h}, M^{s v}$ and $M^{m v}$ which refer to handcarts, vehicles with small and medium size respectively. $I^{c}=\left\{I^{h}, I^{v h}, I^{B}\right\}$ is the set of demand nodes in both day and night shift. More on this explanation nomenclatures and proposed models are mentioned in follow.

\section{Table 1}

Nomenclatures of waste collection routing problem

\begin{tabular}{ll}
\hline notations & definition \\
\hline$d_{i j m}$ & Darameters \\
$c_{i j m}$ & unit cost of transporting from node $i$ to $j(i, j \in V)$ by vehicle of type $m(m \in M)$ \\
$p_{i j m}$ & travel time between nodes $i$ and $j(i, j \in V)$ by vehicle of type $m(m \in M)$ \\
$q_{i}$ & volume loaded in ith node $\left(i \in I^{c}\right)$ \\
$L_{i}$ & lower bound of time window for node $i\left(i \in I^{c} \cup I^{i f}\right)$ \\
$U_{i}$ & upper bound of time window for node $i\left(i \in I^{c} \cup I^{i f}\right)$ \\
$C_{m}^{v}$ & capacity of vehicle of type $m(m \in M)$ \\
$C_{t}^{s}$ & time duration of shift $t(t \in T)$ \\
$P c_{i}$ & penalty cost of not servicing customer $i\left(i \in I^{c}\right)$ \\
$O l c_{i}$ & penalty cost of not servicing customer $i$ in related time window $\left(i \in I^{c}\right)$
\end{tabular}


$v c_{m} \quad$ maintenance cost of vehicle of type $m(m \in M)$

$O c_{m} \quad$ labor cost of vehicle of type $m(m \in M)$

$N_{m d} \quad$ Number of vehicles type $m$ assigned to depot $d(m \in M),\left(d \in I^{D}\right)$

FC cost of recycling and packaging of wastes

Variables

$x_{i j m d t}$

$=1$ if a vehicle of type $m(m \in M)$ travelling along arc $(i, j)(i, j \in V)$ in shift $t$

$(t \in T)$ and originating from depot $d\left(d \in I^{D}\right)$ is selected, and $x_{i j m d t}=0$

otherwise

$z_{i t} \quad=1$ if $i$ th $\left(i \in I^{c}\right)$ node is not serviced in shift $t(t \in T)$

$y_{i j m t} \quad$ volume of waste transferred along arc $(i, j)(i, j \in V)$ by vehicle of type $m$ $(m \in M)$ in shift $t(t \in T)$

$n_{m d} \quad$ number of vehicle of type $m(m \in M)$ assigned to depot $d\left(d \in I^{D}\right)$

$E_{i t} \quad$ Earliness of servicing customer $i\left(i \in I^{c}\right)$ in shift $t(t \in T)$

$T_{i t} \quad$ Tardiness of servicing customer $i\left(i \in I^{c}\right)$ in shift $t(t \in T)$

$A_{i t} \quad$ time of getting served of $i$ th $\left(i \in I^{c}\right)$ node in shift $t(t \in T)$

Model 1:

$$
\begin{aligned}
& \min z=\sum_{m \in M} \sum_{d \in I^{D}} v c_{m} \times n_{m d}+\sum_{(i, j) \in V} \sum_{m \in M} \sum_{t \in T} \sum_{d \in I^{D}} c_{i j m} \times d_{i j m} \times x_{i j m d t}+\sum_{i \in V-\left\{I^{D}\right\}} \sum_{t \in T} O l c_{i}\left(E_{i t}+T_{i t}\right) \\
& +\sum_{i \in I^{c}} \sum_{t \in T} P c_{i} \times z_{i t} \times q_{i}+F C \sum_{i \in I^{c}} \sum_{m \in M} \sum_{d \in I^{d}} \sum_{t \in T} q_{i}\left(1-z_{i t}\right)+\sum_{m \in M} \sum_{d \in I^{D}} O c_{m} \times n_{m d}
\end{aligned}
$$

subject to

$$
\begin{array}{ll}
\sum_{i \in V} \sum_{m \in M} \sum_{d \in I^{D}} x_{i j m d t}=1 & \forall j \in I^{c}, t \in T \\
\sum_{j \in V} \sum_{m \in M} \sum_{d \in I^{D}} x_{i j m d t}=1 & \forall i \in I^{c}, t \in T \\
\sum_{j \in I^{c}} \sum_{t \in T} x_{i j m d t}=n_{m d} & \forall i \in I^{d}, m \in M, d \in I^{D} \\
n_{m d} \leq N_{m d} & \forall m \in M, d \in I^{D} \\
\sum_{i \in V} x_{i j m d t}=\sum_{i \in V} x_{j i m d t} & \forall j \in V, m \in M, d \in I^{D}, t \in T \\
\sum_{j \in\left(I^{c} \cup I^{i f}\right)} y_{i j m t}-\sum_{j \in\left(I^{D} \cup I^{c}\right)} y_{j i m t}=q_{i} \times \sum_{j \in\left(I^{c} \cup I^{i f}\right)} & x_{i j m d t} \\
y_{i j m t} \leq \sum_{d \in I^{D}} C_{m}^{v} \times x_{i j m d t} & \forall i \in I^{c}, m \in M, t \in T, d \in I^{D} \\
A_{j t}=\sum_{i} \sum_{m} \sum_{d}\left(p_{i j m}+A_{i t}\right) \times x_{i j m d t} & \forall i \in V, j \in V, m \in M, t \in T
\end{array}
$$




$$
\begin{array}{ll}
E_{i t}=\max \left\{0, L_{i}-A_{i t}\right\} & \forall i \in V-\left\{I^{D}\right\}, t \in T \\
T_{i t}=\max \left\{0, A_{i t}-U_{i}\right\} & \forall i \in V-\left\{I^{D}\right\}, t \in T \\
x_{i j m d t} \leq M\left(\max \left\{0, \varepsilon-\left(y_{i j m t}-C_{m}^{v}\right)\right\}\right) & \forall i \in V, j \in\left\{I^{v h}, I^{i f}\right\}, m \in M, d \in I^{D}, t \in T \\
C_{t}^{s}-A_{i t}-M\left(1-z_{i t}\right) \leq 0 & \forall i \in V, t \in T \\
\sum_{(i, j) \in I^{h}} \sum_{m \in M-\left\{M^{h}\right\}} x_{i j m d t}=0 & \forall d \in I^{D}, t \in T \\
x_{i j m d 2}=0 & \forall(i, j) \in V-\left\{I^{B}\right\}, m \in M^{h}, d \in I^{D} \\
x_{i j m d t}=0 & \forall i \in I^{D}, j \in\left\{I^{D} \cup I^{i f}\right\} \\
y_{i j m t}=0 & \forall i \in\left\{I^{D} \cup I^{i f}\right\}, j \in V, m \in M, t \in T \\
A_{i t}=0 & \forall i \in I^{D}, t \in T \\
x_{i j m d t} \in\{0,1\} & \forall i \in V, j \in V, m \in M, d \in I^{D}, t \in T \\
z_{i t} \in\{0,1\} & \forall i \in V, t \in T \\
y_{i j m t} \geq 0 & \forall i \in V, t \in T \\
n_{m d} \geq 0 & \forall i \in V, j \in V, m \in M, t \in T \\
E_{i t}, T_{i t}, A_{i t} \geq 0 & \forall i \in M
\end{array}
$$

The objective function (1) minimizes total cost of system which includes maintenance cost of fleet, travelling cost, penalty cost of servicing out of time window and not servicing a customer, labor cost, and recycling and packaging cost respectively. Eqs. (2) guarantee that each customer is served once whereas Eqs. (3) ensure the flow conservation. It means that if a vehicle enters a customer site, must leave. Fleet size assigned to each depot to service a wide region is reached by Eqs. (4) and constraint (5) shows that fleet size cannot exceed the maximum fleet size. In constraints (6) it is shown that only one vehicle type, originated from a depot, serves customers along a route. Volume of wastes loaded along arc $(i, j)$ is depicted in Eqs. (7). Constraints (8) satisfy the capacity limitation of each vehicle. Arrival time to each customer node is calculated by constraints (9). Also, earliness and tardiness in servicing nodes regards to the related time window are shown in constraints (10) and (11) respectively. In constraints (12) full-loaded vehicles travel to disposal site, completing their route. Inequalities (13) show that in case of not completing collection activities during a shift, some of customers are not served which leads to increase the cost of system in terms of penalty cost. Constraints (14) show that nodes with $\alpha_{i} \leq 0.3$ would be served only by handcarts. As mentioned before in second shift handcarts are not used. This is seen in Eqs. (15). Obviously, there is no path from depots to disposal sites as shown in Eqs. (16). The vehicle departed from a depot or disposal facility is empty. This can be reached by constraints (17) and based on originating a route from depot; constraints (18) demonstrate that the arrival time to each depot is zeros. Constraints (19), (20) refer to binary variables whereas constraints (21), (22) and (23) ensure the non-negativity of variables.

In second stage, waste packs are provided to be dispatched to the rail stations by Containers. Let directed graph $G(V, E, M) . V$ is a set of disposal sites, nodes traversed by containers, specified loading 
rail stations, nodes traversed by train and final destination which are demonstrated as $V^{i f}, V^{h}, V^{h r}, V^{r}, T$ respectively. $E=\left\{E_{h}, E_{r}\right\}$ is a set of arcs between two nodes and is divided into two subsets, road paths and rail paths. According to the implementation of two different transit systems, $M$ refers to $M^{l v}$ as container and $M^{t}$ as train. The considered graph is formulated as a multi-objective problem to minimize costs and destructive effects of waste transportation on environment, concurrently. For minimizing environmental effects of transportation, authors discuss two qualitative criteria; probability of accident in each arc and emitted pollution rate from transportation vehicles. Hence, it is attempted to route vehicles optimally in order to decrease the environmental withering impacts of transportation activities. The nomenclatures and proposed model of this stage are as below.

Table 2

Nomenclatures of multimodal routing problem

\begin{tabular}{|c|c|}
\hline notations & definition \\
\hline $\begin{array}{l}\text { Parameters } \\
d_{i j m}\end{array}$ & $\begin{array}{l}\text { distance between nodes } i \text { and } j(i, j \in V) \text { travelled by transit system of type } m \\
(m \in M)\end{array}$ \\
\hline$p_{i j m}$ & travel time from node $i$ to $j(i, j \in V)$ by transit system of type $m(m \in M)$ \\
\hline$L_{i}$ & lower bound of time window related to node $i\left(i \in V^{h r}\right)$ \\
\hline$U_{i}$ & upper bound of time window related to node $i\left(i \in V^{h r}\right)$ \\
\hline$C_{m}^{v}$ & capacity of vehicle of type $m(m \in M)$ \\
\hline$S_{i j}$ & unit cost of transporting from node $i$ to $j$ by train $\left(i, j \in V^{R}\right)$ \\
\hline$C_{i j}$ & unit cost of transporting from node $i$ to $j$ by trucks $\left(i, j \in V^{T}\right)$ \\
\hline$v c_{m}$ & maintenance cost of transit system of type $m(m \in M)$ \\
\hline$O c_{m}$ & labor cost of transit system of type $m(m \in M)$ \\
\hline Tc & loading cost of train \\
\hline$\beta_{e}$ & probability of accident in arc $e(e \in E)$ \\
\hline$\gamma_{m}$ & emission pollution rate of transit system of type $m(m \in M)$ \\
\hline$c$ & volume of waste pack \\
\hline$T w_{i}$ & volume of total waste should be packed in disposal facility $i$ \\
\hline$N_{m d}$ & Number of vehicles type $m$ assigned to depot $d(m \in M),\left(d \in I^{D}\right)$ \\
\hline Olc $c_{i}$ & penalty cost of not servicing customer $i$ in related time window $i \in\left\{V^{h}, V^{h r}, V^{r}\right\}$ \\
\hline \multicolumn{2}{|l|}{ Variables } \\
\hline$X_{i j m d}$ & $\begin{array}{l}=1 \text { if a vehicle of type } m(m \in M) \text { travelling along arc }(i, j)(i, j \in V) \text { and } \\
\text { originating from depot } d\left(d \in V^{i f} \cup V^{h r}\right)\end{array}$ \\
\hline$Q_{i p}$ & $\begin{array}{l}\text { number of waste packs transferred from interfacility } i\left(i \in V^{i f}\right) \text { to train loading } \\
\text { station } p\left(p \in V^{h r}\right)\end{array}$ \\
\hline$n_{m d}$ & number of vehicles of type $m(m \in M)$ assigned to depot $d\left(d \in V^{i f} \cup V^{h r}\right)$ \\
\hline$E_{i}$ & Earliness of loading in rail station $i$ \\
\hline$T_{i}$ & Tardiness of loading in rail station $i$ \\
\hline$A_{i}$ & Arrival time to node $i$ by desired vehicle \\
\hline
\end{tabular}


Model 2:

$$
\begin{aligned}
& \min z_{1}=\sum_{j \in V^{h r}} \sum_{i \in V^{i f}} T c \times Q_{i j}+\sum_{(i, j) \in\left\{V^{T}\right\}} \sum_{d \in V^{i f}} c_{i j} \times d_{i j M^{l v}} \times x_{i j M^{l v d}}+\sum_{(i, j) \in\left\{V^{R}\right\}} \sum_{d \in V^{h r}} s_{i j} \times d_{i j M^{t}} \times x_{i j M^{t} d} \\
& +\sum_{i \in V^{h r}} \operatorname{Olc}_{i}\left(E_{i}+T_{i}\right)+\sum_{m \in M} \sum_{d \in\left\{V^{i f} \cup V^{h r}\right\}} v c_{m} \times n_{m d}+\sum_{m \in M} \sum_{d \in\left\{V^{i f} \cup V^{h r}\right\}} O c_{m} \times n_{m d}
\end{aligned}
$$

$$
\min z_{2}=\sum_{(i, j) \in V} \sum_{m \in M} \sum_{e \in E} \sum_{d \in\left\{V^{i f} \cup V^{h r}\right\}} \beta_{e} \times x_{i j m d}+\sum_{(i, j) \in V} \sum_{m \in M} \sum_{d \in\left\{V^{i f} \cup V^{h r}\right\}} \gamma_{m} \times x_{i j m d}
$$

subject to

$$
\begin{aligned}
& \sum_{i \in V} \sum_{m \in M} \sum_{d \in\left(V^{i f} \cup V^{h r}\right)} x_{i j m d}=1 \\
& \forall j \in\left\{V^{h} \cup V^{r}\right\} \\
& \sum_{j \in V} \sum_{m \in M} \sum_{d \in\left(V^{i f} \cup V^{h r}\right)} x_{i j m d}=1 \\
& \forall i \in\left\{V^{h} \cup V^{r}\right\} \\
& \sum_{j \in V^{h}} X_{i j m d}=n_{m d} \\
& \forall i \in V^{i f}, m \in M, d \in\left\{V^{i f} \cup V^{h r}\right\} \\
& n_{m d} \leq N_{m d} \\
& \forall m \in M, d \in I^{D} \\
& \sum_{i \in V} X_{i j m d}=\sum_{i \in V} x_{j i m d} \\
& \forall j \in V, m \in M, d \in\left(V^{i f} \cup V^{h r}\right) \\
& \sum_{p \in V^{h r}} Q_{j p} \leq \frac{T w_{j}}{C} \\
& \forall j \in V^{i f} \\
& Q_{i p} \leq C_{M^{l v}}^{v} \\
& \forall i \in V^{i f}, p \in V^{h r} \\
& A_{j}=\sum_{i \in V} \sum_{m \in M} \sum_{d \in\left\{V^{i f} \cup V^{h r}\right\}}\left(p_{i j m}+A_{i}\right) \times x_{i j m d} \\
& \forall j \in V \\
& E_{i}=\max \left\{0, L_{i}-A_{i}\right\} \\
& \forall i \in V^{h r} \\
& T_{i}=\max \left\{0, A_{i}-U_{i}\right\} \\
& x_{i j m d} \in\{0,1\} \\
& \forall i \in V^{h r} \\
& n_{m d}>0 \\
& \forall i \in V, j \in V, m \in M, d \in V^{i f} \\
& \forall m \in M, d \in V^{i f} \\
& Q_{j p} \geq 0 \\
& \forall i \in V^{i f}, p \in V^{h r} \\
& E_{i}, T_{i}, A_{i} \geq 0 \\
& \forall i \in V
\end{aligned}
$$

In second stage the cost factor and environmental factors are considered concurrently. By considering first objective function (24), total cost of system is minimized. In this regard loading cost of train, travelling cost, and penalty cost of loading out of time window are minimized. On the other hand, second objective function (25) takes into account emitted pollution rate and environmental destructive effects of waste transportation activities. Constraints (26)-(30) are similar to constraints (2)-(6) explained before. Constraints (31) calculate the number of waste packs transferred from a disposal facility to rail loading stations. These values are proportional to volume of wastes collected from customer nodes. Capacity of containers is satisfied by inequalities (32). Arrival time to each node by desired transfer system is shown in Eqs. (33). Earliness and tardiness value of loading in rail stations are shown by constraints (34) and (35) respectively. Constraints (36) refer to binary variables and 
constraints (37), (38) and (39) refer to non-negative variables. Obviously the considered quantitative and qualitative factors have no contradiction and minimization of cost factors results in minimization of qualitative factors. Given the difference scale of both objective functions, the objective function is normalized and integrated in Eq. (40).

$$
\begin{aligned}
& \min z=\frac{\sum_{j \in V^{h r}} \sum_{i \in V^{f f}} T c \times Q_{i j}}{\max \left(\sum_{j \in V^{h r}} \sum_{i \in V^{f f}} T c \times Q_{i j}\right)}+\frac{\sum_{(i, j) \in\left\{V^{T}\right.} \sum_{d \in V^{i f}} c_{i j} \times d_{i j M^{l v}} \times x_{i j M^{l v} d}}{\max \left(\sum_{(i, j) \in\left\{V^{T}\right\}} \sum_{d \in V^{i f}} c_{i j} \times d_{i j M^{l v}} \times x_{i j M^{l v} d}\right)}+\frac{\sum_{(i, j) \in\left\{V^{R}\right\}} \sum_{d \in V^{h r}} s_{i j} \times d_{i j M^{t}} \times x_{i j M^{t} d}}{\max \left(\sum_{(i, j) \in\left\{V^{R}\right\}} \sum_{d \in V^{h r}} s_{i j} \times d_{i j M^{t}} \times x_{i j M^{t} d}\right)} \\
& +\frac{\sum_{i \in V^{h r}} O \operatorname{Ol}_{i}\left(E_{i}+T_{i}\right)}{\max \left(\sum_{i \in V^{h r}} O \operatorname{Ol}_{i}\left(E_{i}+T_{i}\right)\right)}+\frac{\sum_{m \in M} \sum_{d \in\left\{V^{i f} \cup V^{h r}\right\}} v c_{m} \times n_{m d}}{\max \left(\sum_{m \in M} \sum_{d \in\left\{V^{i f} \cup V^{h r}\right\}} v c_{m} \times n_{m d}\right)}+\frac{\sum_{m \in M} \sum_{d \in\left\{V^{i f} \cup V^{h r}\right\}} O c_{m} \times n_{m d}}{\max \left(\sum_{m \in M} \sum_{d \in\left\{V^{i f} \cup V^{h r}\right\}} O c_{m} \times n_{m d}\right)} \\
& +\sum_{(i, j) \in V} \sum_{m \in M} \sum_{e \in E} \sum_{d \in\left\{V^{i f} \cup V^{h r}\right\}} \beta_{e} \times x_{i j m d}+\sum_{(i, j) \in V} \sum_{m \in M} \sum_{d \in\left\{V^{i f} \cup V^{h r}\right\}} \gamma_{m} \times x_{i j m d}
\end{aligned}
$$

For transportation system area (See Kuo et al., 2012; Liu, 2013; Chen et al., 2011; Xiong et al., 2012), we apply an improved genetic algorithm which is a hybridization of genetic algorithm and a heuristic algorithm. In order to validate applicability of the developed algorithms, results of the algorithms are compared with those of GAMS for some small-size problem instances. The overview of our approach is briefly given as below.

\subsection{Genetic algorithm}

Genetic algorithm is a destructive and improving heuristic method to optimize large scale problems which was first introduced by Holland (1975). The algorithm inspired by nature improves fitness function of chromosomes or, in another words, improves obtained solutions. Based on the algorithm and natural genetic structure, shifting from one generation to another is resulted during 4 stages: parent selection, alteration, evaluating fitness function and substitute. In parent selection stage, desired parents to reproduction the new generation are determined. Alteration operators of algorithm consist of crossover and mutation modes. Fitness function of new generation determines competency of results to reproduction of next generation. By considering fitness function in substitution stage, desired parents are altered. In this study Taguchi method is applied to increase the speed of algorithm by setting parameters appropriately. In the proposed study, the classic genetic algorithm is hybridized with a heuristic algorithm which improves the quality of obtained results. The steps of developed algorithm are explained below.

\section{Step 1. Clustering}

Because of multiple depots from which vehicles operate, the demand nodes are clustered and the proposed algorithm is applied for each subset. The implemented clustering approach is a variant of dividing-based method that cluster nodes based on a target.

\section{Step 2. Initial Population}

The basis of the proposed heuristic algorithm is inspired from Benjamin et al. (2013). In this step, Permutation representation is selected to illustrate the chromosomes in which each gene represents the destination of travel. Initial route is designed by following below stages.

Step 2.1: Set $\mathrm{B}=\mathrm{C}$ ( $\mathrm{C}$ is a set of each cluster demand nodes).

Step 2.2: Randomly select a node from set $B$ and start the tour. Eliminate selected node from B and add it to $F$ ( $F$ is a set of served nodes). Calculate the value of servicing time and remaining capacity. 
Step 2.3: If the service time and capacity constraints are satisfied, put the nodes which have joint time window in set $\mathrm{T}$ ( $\mathrm{T}$ is a set of nodes that might be destination of next travel) and go to step 2.5; otherwise go to step 2.4 .

Step 2.4: In this step two cases are possible.

Case 1: If the capacity constraint is violated, vehicle travels to a disposal site assigned to cluster. In case of having enough time, route another trip to collect wastes. In this regard, direct the vehicle to the nearest neighbor node that has desired time window related to service time. Eliminate the node from $B$ and put it in $F$. Moreover, update the remaining capacity and service time parameters. If there is no enough time, complete the tour through going back to the depot. Go to step 2.6.

Case 2: If the shift time is violated, in case that the recent node is a demand node, direct the vehicle to disposal site and go back to depot and complete the tour. Otherwise, if the recent node is a disposal site, go back to the depot and go to step 2.6.

Step 2.5: In this stage, two cases is possible:

Case 1: Randomly select a node from set $T$. If the selected node violates the capacity and service time constraint, select another one. Eliminate the node from $B$ and add it to $F$. Calculate the remaining capacity and service time parameters and go to step 2.3.

Case 2: If $T$ is empty, select a node from $B$ which has the least distance from the recent node. Go to step 2.3.

Step 2.6. If the recent node points out a depot and B is not empty, considering fleet size limitation, route another vehicle. Go to step 2.7.

Step 2.7. If the stop criteria determined by Taguchi method is reached, stop. (Initial population parameter $=100$ ).

\section{Step 3: Parent selection}

In this step, according to the fitness function, parents are selected to reproduction process. Here a roulette wheel is applied to determine the probability of selecting each chromosome as parent. This value is calculated by the following equation,

$$
p=e^{\left(\left(-\beta^{*} \cos t\right) / \text { worst } \cos t\right)}
$$

where $\beta$ is the parameter of roulette wheel which is set to 9. The roulette wheel's parts indicate the cumulative sum of probabilities. By creating a random number in $[0,1]$, desired parents to reproduction process are selected. Hence, obviously as fitness function increases, probability of selection increase.

\section{Step 4. Crossover}

Some genes of chromosomes of both parents are selected to alter. Number of selected parents for crossover is a parameter which is determined by Taguchi method (Crossover parameter=0.7). In order to satisfy the capacity and service time constraint, an edge crossover structure is implemented to merge and improve the solutions. The considered edge table depicts the genes adjacent to each node. In this step, an improved form of classic edge crossover structure is applied. According to VRP assumptions, each vehicle starts the tour from depot and travel to a random node. The adjacent node which has the least adjacency length is the destination of next travel. Considering capacity and service time constraints result in two cases similar to step 2.4 of initial population. 
This operation generates a new chromosome from one parent. There are different types of mutation operators e.g. swap mutation, insert mutation, scramble mutation, and inversion mutation. In this study regard to discussed problem and permutation representation of results swap mutation is applied (Mutation parameter=0.4). Swap mutation replaces two random genes of chromosome and creates new chromosome. Figure 1 demonstrates operation of this operator.

\begin{tabular}{|c|c|c|c|c|c|c|c|c|}
\hline 1 & 7 & 9 & 3 & 5 & 6 & 4 & 2 & 8 \\
\hline 1 & 2 & 9 & 3 & 5 & 6 & 4 & 7 & 8 \\
\hline
\end{tabular}

Fig 1. Swap mutation operator

Step 6. Heuristic improvement operator

Regards to the importance of discussed problem and its applicability in real world, in this stage a heuristic phase is designed to improve the quality of solutions. The process is based on two approaches:

Approach 1: In contrast to mutation, in this stage nodes of different routes are swapped. So that, for a random node, all adjacent nodes with joint time window is determined. If there is a node served during other routes, considering capacity and service time, exchange the nodes.

Approach 2: The main objective of this approach is decreasing the number of vehicles. In this regard, in case of satisfying all constraints, nodes served by last vehicle will be allocated to other routes. Fleet size minimization results in reduction of time and cost expend to collect wastes.

\section{Step 7. Evaluating fitness function}

Finally, new generation is selected among initial population and new chromosomes are derived from crossover, mutation and heuristic improvement operations. Chromosomes with high fitness function are selected as parents for next generation production.

\section{Step 8. Stop criteria}

At the end of each repetition, if stop criteria is reached, the algorithm stops. Here, it is considered that after some repetitions, algorithm stops. Based on Taguchi method, the stop criteria is set to 400 repetitions. It must be noted that the proposed algorithm is applicable for rail/road transportation system by making some changes. Although the speed of road transit system is high, but the safety and threatening environmental effects of rail transit system is more. Hence, it is better to design a route with less pollution and accident rate that increases the safety of transportation. During solving the multimodal transportation model, instead of capacity and service time parameters, pollution rate and accident risk are considered. For the second model, after directing wastes to a rail station, a rail network is routed to transfer wastes to final destination. For this model, in case that waste volume exceeds the train capacity, the heuristic improvement phase is applicable.

\section{Computational results}

Considering the importance of waste collection system in each municipality and the authorities need to an efficient scheduling, in this study a mathematical model and solution methodology are proposed taking the advantages of multimodal transportation systems. As mentioned before, in order to enhance 
the quality of obtained solutions, heuristic and genetic algorithms are hybridized. To evaluate the validity of the proposed structure and applicability of implemented algorithms, several instances in small, medium and large sizes are tested. The scales of examples are illustrated in Table 3.

Table 3

Scales of examples

\begin{tabular}{|c|c|c|c|c|c|c|c|}
\hline & example & Nodes & Depot & waste bins & $\begin{array}{c}\text { Nodes serviced by } \\
\text { handcarts }\end{array}$ & $\begin{array}{c}\text { Nodes serviced by } \\
\text { vehicles }\end{array}$ & $\begin{array}{l}\text { disposal } \\
\text { facility }\end{array}$ \\
\hline \multirow{11}{*}{ 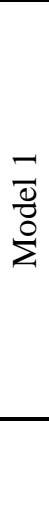 } & 1 & 13 & 2 & 2 & 2 & 5 & 2 \\
\hline & 2 & 22 & 2 & 3 & 4 & 10 & 3 \\
\hline & 3 & 29 & 2 & 5 & 5 & 15 & 2 \\
\hline & 4 & 41 & 3 & 5 & 10 & 20 & 3 \\
\hline & 5 & 62 & 4 & 10 & 15 & 30 & 3 \\
\hline & 6 & 80 & 4 & 12 & 25 & 35 & 4 \\
\hline & 7 & 127 & 4 & 20 & 40 & 60 & 3 \\
\hline & 8 & 183 & 4 & 25 & 50 & 100 & 4 \\
\hline & 9 & 224 & 5 & 35 & 70 & 110 & 4 \\
\hline & 10 & 375 & 5 & 50 & 100 & 215 & 5 \\
\hline & example & Nodes & Depot & $\begin{array}{c}\text { loading rail } \\
\text { stations }\end{array}$ & $\begin{array}{c}\text { Nodes traversed } \\
\text { by containers }\end{array}$ & $\begin{array}{c}\text { Nodes traversed } \\
\text { by train }\end{array}$ & $\begin{array}{c}\text { Final } \\
\text { destination }\end{array}$ \\
\hline \multirow{10}{*}{ 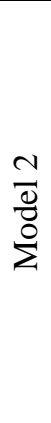 } & 1 & 15 & 2 & 3 & 5 & 4 & 1 \\
\hline & 2 & 29 & 3 & 3 & 16 & 6 & 1 \\
\hline & 3 & 37 & 2 & 3 & 25 & 6 & 1 \\
\hline & 4 & 42 & 3 & 3 & 30 & 5 & 1 \\
\hline & 5 & 53 & 3 & 4 & 40 & 5 & 1 \\
\hline & 6 & 55 & 4 & 4 & 40 & 6 & 1 \\
\hline & 7 & 64 & 3 & 4 & 50 & 6 & 1 \\
\hline & 8 & 72 & 4 & 5 & 55 & 7 & 1 \\
\hline & 9 & 81 & 4 & 6 & 60 & 9 & 2 \\
\hline & 10 & 86 & 5 & 8 & 70 & 10 & 2 \\
\hline
\end{tabular}

Defined parameters for both models are generated randomly which follow uniform distribution function specified in Table 4 and Table 5.

Table 4

Distribution function of nominal data for model 1

\begin{tabular}{llllll}
\hline Parameter & $d_{i j m}$ & $p_{i j m}$ & $p p_{i}$ & $p t_{i}$ & $q_{i}$ \\
Interval & $(0.001,15)$ & $(1,70)$ & $(1,5)$ & $(10,20)$ & $(3,80)$ \\
Parameter & $L_{i}$ & $U_{i}$ & $C_{m}^{v}$ & $c_{i j m}$ & $P c_{i}$ \\
Interval & $(0,15)$ & $(15,30)$ & $(100,1000)$ & $(200,1300)$ & $(800,2500)$ \\
Parameter & Olc $c_{i}$ & $v c_{m}$ & $O c_{m}$ & $F C$ & $N_{m d}$ \\
Interval & $(1000,2200)$ & $(1000,2000)$ & $(20000,35000)$ & $(17000,22000)$ & $(1,5)$ \\
\hline
\end{tabular}

The reason behind diversity in values of some parameters is considering width of passageways and implementing both handcarts and vehicles.

Table 5

Distribution function of nominal data for model 2

\begin{tabular}{llllll}
\hline Parameter & $d_{i j m}$ & $p_{i j m}$ & $L_{i}$ & $U_{i}$ & $C_{m}^{v}$ \\
Interval & $(100,600)$ & $(45,75)$ & $(0,30)$ & $(30,60)$ & $(25,110)$ \\
Parameter & $S_{i j}$ & $c_{i j}$ & $v c_{m}$ & $O c_{m}$ & $T c$ \\
Interval & $(10000,16000)$ & $(7000,12000)$ & $(2000,12000)$ & $(25000,35000)$ & $(3000,6500)$ \\
Parameter & $\beta_{e}$ & $\gamma_{m}$ & $O l c_{i}$ & $T w_{i}$ & $N_{m d}$ \\
Interval & $(0,1)$ & $(0,1)$ & $(2000,5000)$ & $(300,450)$ & $(1,3)$ \\
\hline
\end{tabular}


Based on the generated parameters, the models are solved by genetic algorithm. Also to justify the validation of proposed algorithm, the results of small size instances are compared to those of GAMS software. Summary of test results obtained by implementing GAMS software and genetic algorithm are provided in Table 6.

\section{Table 6}

System cost of samples

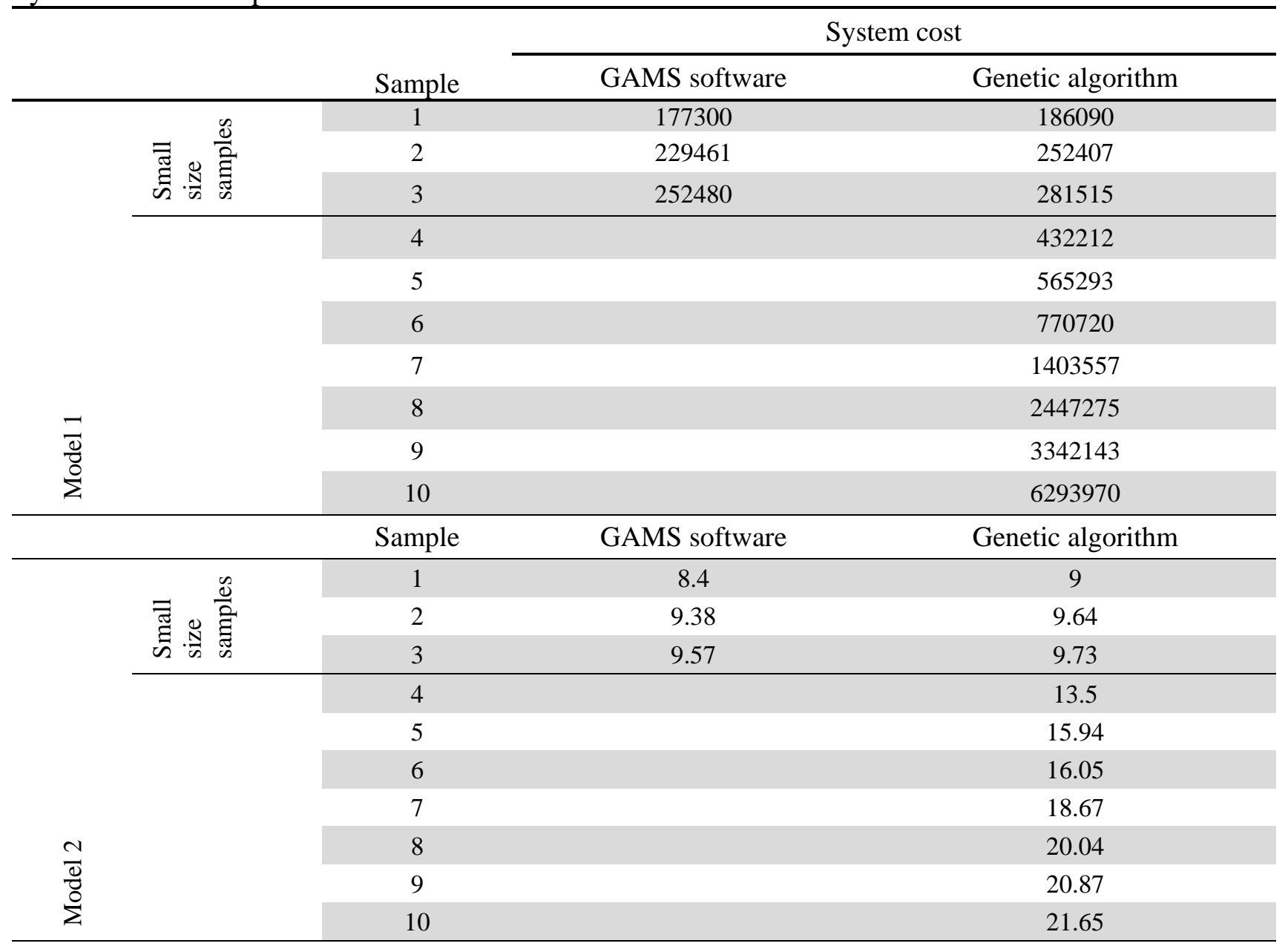

Comparison of results shows that genetic algorithm has about 4\%-10\% diversification from GAMS results based on which authors claim that the solution approach is an efficient method to solve the models. For an example, consider sample 1 of both models. As it is assumed in Table 3, a nonhomogeneous collection fleet operates from two dispersed depot. According to recorded data, $\alpha_{i}$ is determined for all demand nodes based on which two nodes must be served by handcarts. Fig. 2 depicts the obtained route of both genetic algorithm and GAMS software.

Obviously only one vehicle type serves each node in one visit and flow conservation is satisfied. At the end of shift, each vehicle travels to disposal facility to unload wastes and come back to depot. In disposal facility solid waste packs are loaded by containers to convey to loading rail stations. Proposed multimodal transportation system leads to minimize the environmental destructive effects of waste transportation by reduction of transportation time and distance. On the other hand by developing this system accessibility to nodes increases in terms of taking advantage of different transit systems. Cooperating the advantages of both road and rail transportation system, provides a safe system to transport waste from houses to factories. 


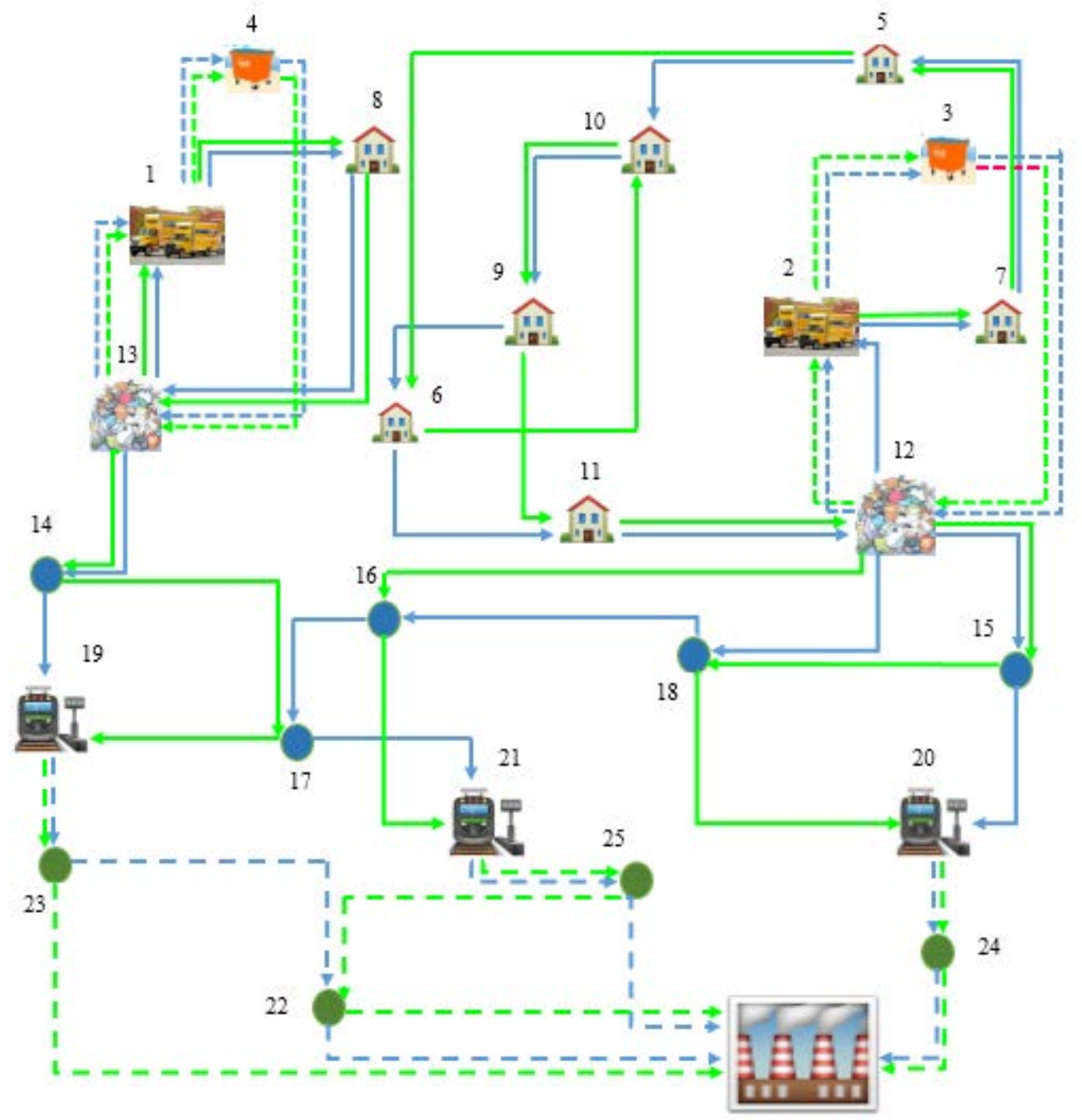

Result of GAMS

Result of Genetic algorithm

Routes in second shift

$\longrightarrow$ Routes in first shift and intercity

$-\rightarrow$ Routes of trains

Fig. 2. Routing of multimodal transportation vehicles (sample 1)

As it is supposed in provided study, rail loading stations must be selected within stations through which few passengers traverse. The reason is health of environment and total time of travelling. Due to decaying wastes during long time, travel time is an important parameter in routing problem. The developed multimodal system decreases the travel time whereas implements few vehicles. In this study the aim of minimizing fleet size is achieved through routing optimally. All other variables can be calculated based on provided routing in Fig. 2. Convergence of algorithm for both models is represented in Fig. 3 and Fig. 4. 


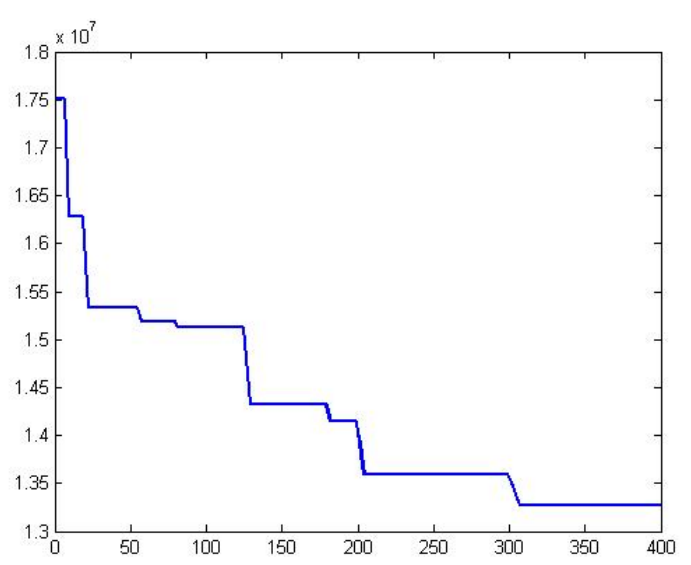

Fig. 3. Convergence of algorithm for model 1

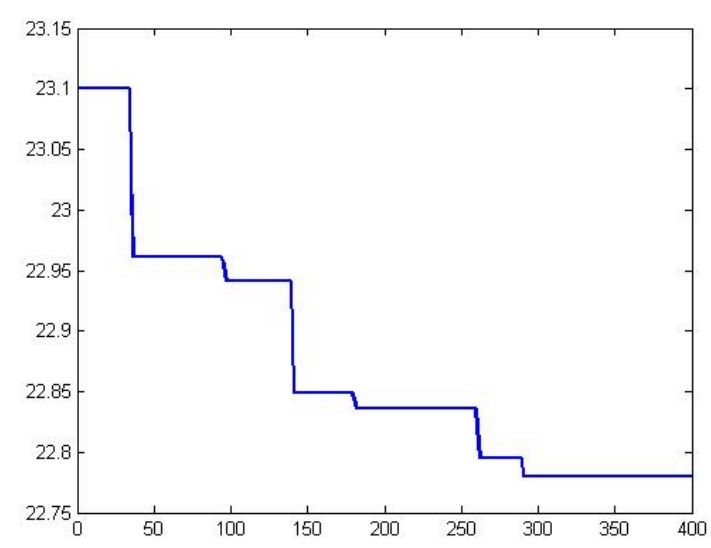

Fig. 4. Convergence of algorithm for model 2

\section{Discussion}

Developed algorithm in section 4, takes advantages of both genetic algorithm operators and a heuristic algorithm to obtain near optimal solutions. In this section sensitivity analysis is provided for both models based on which the sensitivity of system cost to each component is specified. The results of sensitivity analysis help the associated managers consider the most effective factor and analyze it. In waste collection routing problem discussed in model 1, cost is affected by maintenance cost of vehicles, travelling cost, and penalty cost of not servicing of demand nodes or servicing out of related time window, packing of wastes and labor cost. Table 7 refers to impact of each cost component on total system cost of samples 3, 5, 9 .

\section{Table 7}

Sensitivity analysis of model 1 system cost

\begin{tabular}{|c|c|c|c|c|}
\hline \multirow[b]{2}{*}{ Cost factor } & \multicolumn{4}{|c|}{ System cost } \\
\hline & deviation & sample 3 & sample 5 & sample 9 \\
\hline \multirow{4}{*}{ Maintenance cost } & $+50 \%$ & $0.9 \%$ & $2.01 \%$ & $3.6 \%$ \\
\hline & $+25 \%$ & $0.4 \%$ & $1.4 \%$ & $2.1 \%$ \\
\hline & $-25 \%$ & $-0.3 \%$ & $-1.5 \%$ & $-1.98 \%$ \\
\hline & $-50 \%$ & $-0.8 \%$ & $-2 \%$ & $-3.2 \%$ \\
\hline Cost factor & deviation & sample 3 & sample 5 & sample 9 \\
\hline \multirow{4}{*}{ travelling cost } & $+50 \%$ & $23 \%$ & $31.56 \%$ & $38.14 \%$ \\
\hline & $+25 \%$ & $12.3 \%$ & $16.18 \%$ & $18.75 \%$ \\
\hline & $-25 \%$ & $-11.9 \%$ & $-15.98 \%$ & $-17.18 \%$ \\
\hline & $-50 \%$ & $-24 \%$ & $-31.16 \%$ & $-37.93 \%$ \\
\hline Cost factor & deviation & sample 3 & sample 5 & sample 9 \\
\hline \multirow{4}{*}{$\begin{array}{l}\text { penalty cost of servicing } \\
\text { out of time window }\end{array}$} & $+50 \%$ & $2.3 \%$ & $10.34 \%$ & $17.27 \%$ \\
\hline & $+25 \%$ & $1 \%$ & $4.7 \%$ & $6.34 \%$ \\
\hline & $-25 \%$ & $-0.98 \%$ & $-2.54 \%$ & $-9.13 \%$ \\
\hline & $-50 \%$ & $-2.1 \%$ & $-5.28 \%$ & $-15.8 \%$ \\
\hline Cost factor & deviation & sample 3 & sample 5 & sample 9 \\
\hline \multirow{4}{*}{$\begin{array}{l}\text { penalty cost of not } \\
\text { servicing demand node }\end{array}$} & $+50 \%$ & $0 \%$ & $0 \%$ & $3.42 \%$ \\
\hline & $+25 \%$ & $0 \%$ & $0 \%$ & $2.36 \%$ \\
\hline & $-25 \%$ & $0 \%$ & $0 \%$ & $-1.98 \%$ \\
\hline & $-50 \%$ & $0 \%$ & $0 \%$ & $-2.65 \%$ \\
\hline Cost factor & deviation & sample 3 & sample 5 & sample 9 \\
\hline \multirow{4}{*}{$\begin{array}{l}\text { recycling and packing of } \\
\text { solid waste }\end{array}$} & $+50 \%$ & $10.14 \%$ & $11.78 \%$ & $13.67 \%$ \\
\hline & $+25 \%$ & $6.05 \%$ & $6.2 \%$ & $8.67 \%$ \\
\hline & $-25 \%$ & $-5 \%$ & $-5.53 \%$ & $-7.3 \%$ \\
\hline & $-50 \%$ & $-9.2 \%$ & $-11.97 \%$ & $-12.8 \%$ \\
\hline Cost factor & deviation & sample 3 & sample 5 & sample 9 \\
\hline \multirow{4}{*}{ labor cost } & $+50 \%$ & $12.3 \%$ & $13.4 \%$ & $15.29 \%$ \\
\hline & $+25 \%$ & $5.62 \%$ & $6.34 \%$ & $7.28 \%$ \\
\hline & $-25 \%$ & $-3.91 \%$ & $-5.29 \%$ & $-8.76 \%$ \\
\hline & $-50 \%$ & $-11.27 \%$ & $-12.16 \%$ & $-14.78 \%$ \\
\hline
\end{tabular}


Obviously, all cost components influence directly on the total system cost. Total system cost is mostly sensitive to travelling cost rather than others. The reason is that travelling cost has influence on all cost components and results in large deviation. Also labor cost affects system cost noticeably. Due to importance of waste management recently, more attentions and large expenditures are spent to routing vehicles optimally. Sensitivity of system cost to these components is not ignorable and necessitates a systematic plan to control the system. Regards to the similarity of second model's structure to the first one, the sensitivity approach is the same and to the sake of brevity it is not mentioned. Capacity and travel time are other essential factors in routing. Fig. 5, Fig. 6 and Fig. 7 depict the sensitivity of total cost to these parameters.

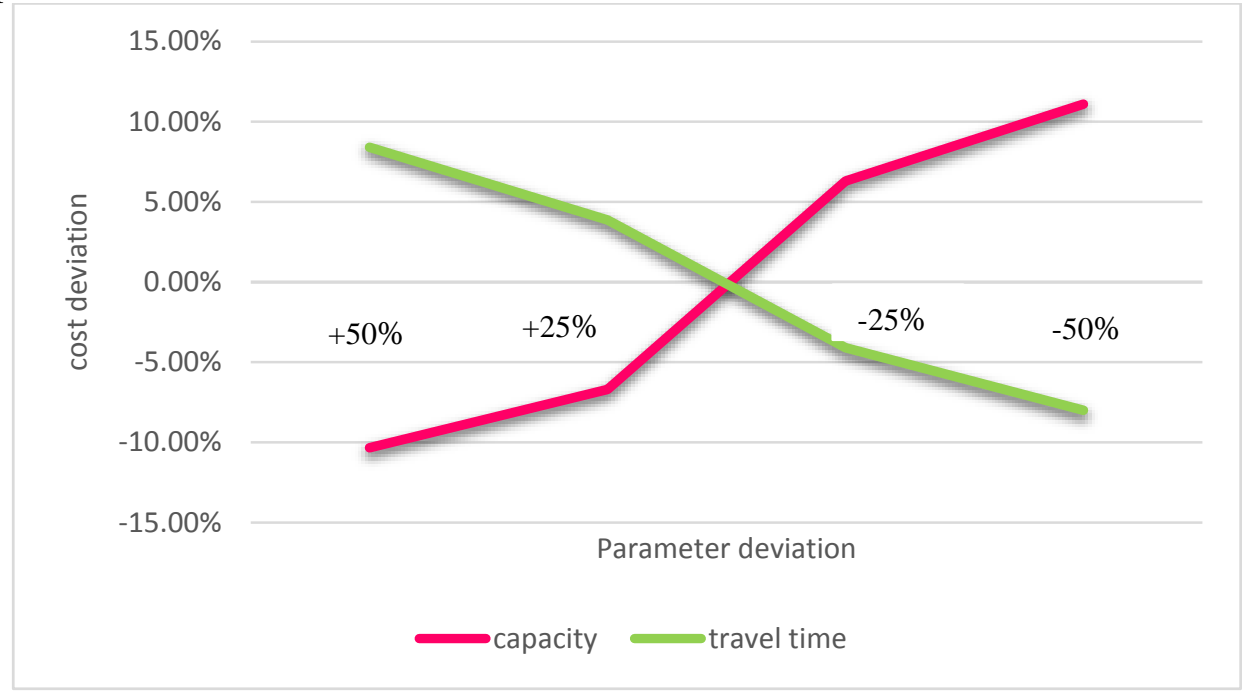

Fig 5. Sensitivity analysis of capacity and travel time for sample 3

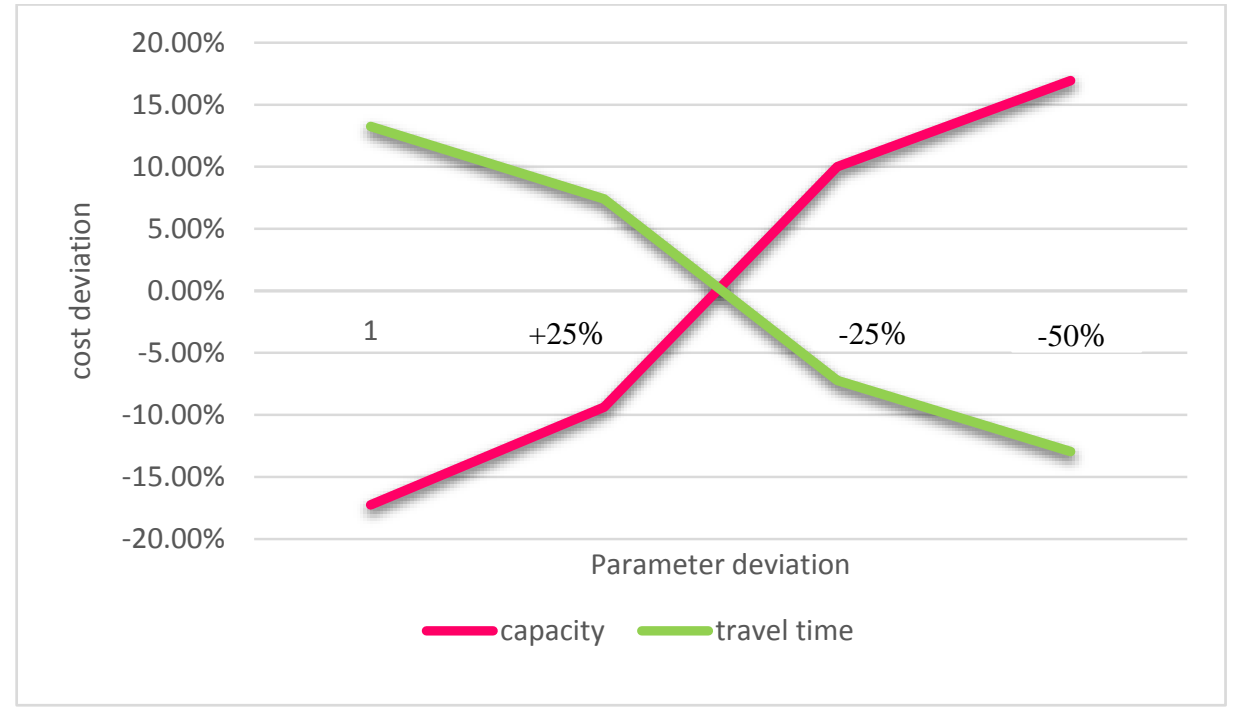

Fig 6. Sensitivity analysis of capacity and travel time for sample 5 


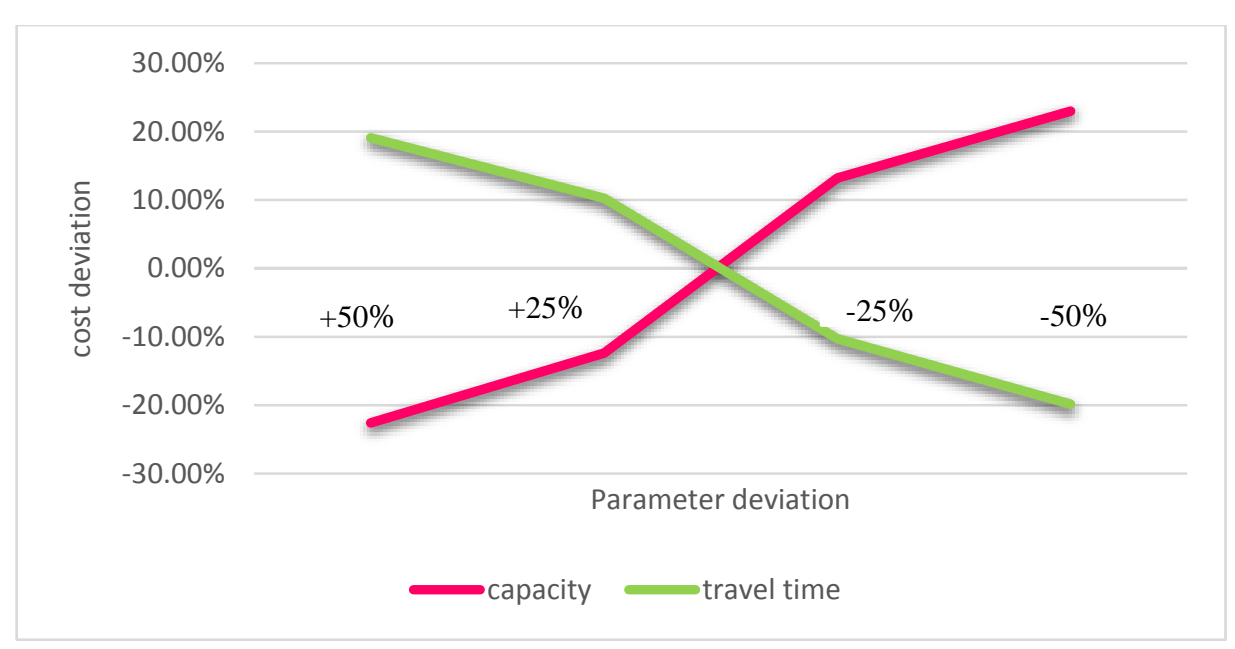

Fig 7. Sensitivity analysis of capacity and travel time for sample 9

In contrast to cost components, capacity parameter affects the total cost inversely. The reason is that by increasing the capacity, fleet size decreases and it influences different costs and routes. However, the impact of travel time is direct. To prove the efficiency of proposed multimodal transportation system rather than single-model transportation system, Table 8 illustrates the objective function of second model for qualitative factors in case that one type vehicle is implemented and compares it with those of multimodal system.

\section{Table 8}

Sensitivity analysis of model 2 system cost

\begin{tabular}{lll}
\hline & GAMS & GA \\
\hline multimodal transportation & 7.57 & 7.75 \\
single-model transportation & 8.81 & 9.39 \\
\hline
\end{tabular}

Obviously, taking advantage of a multimodal transportation system has a great impact on environmental factors and in long-term decreases the adverse effects of waste transportation oh environment.

\section{Conclusion and future research directions}

Nowadays, by considering inevitability of waste production in urban area, municipalities spend large cost on waste collection service. VRP is a fundamental operational problem. Recently, in order to importance of WM, cooperating the VRP and WM has been attracted by researchers. In this study, a hierarchical structure is developed for residential waste collection by taking advantage of two rail and road transit systems. In the discussed routing problem wastes are collected in two shifts. In the first one, wastes are collected from houses whereas in second one only waste bins located in streets are served. Respect to extension of considered area, multiple depots and multiple disposal facilities are considered to cover most regions. A nonhomogeneous fleet including handcarts and vehicles with small and medium sizes start their trip from a depot and after transporting collected wastes to disposal facilities, return to depot from which originate. A multimodal transportation system is implemented to transport wastes more safe and in a short time. Applying such system results in increment of nodes accessibility through different route types. Waste packs are directed to final destination by containers and trains respectively. An improved genetic algorithm is implemented to solve the NP hard models. Comparison of obtained near optimal solutions with those of GAMS validates the models. The applicability of algorithm in generating near optimal solutions for proposed NP hard models is resulted from Table 6. 
Future work will focus on collecting wastes separately. Despite charging fleet cost, it decreases separating cost in disposal facilities. On the other hand, in reality there are some nodes need more visits to collect. Traffic regulation is another limitation in vehicle routing problems which is not considered in this study. Some forbidden turns or one way paths are challenge in residential waste collection routing problems. These regulations limit the accessibility of nodes and violate the complete network characteristics. Transfer graph is another approach applicable in solving multimodal transportation system, implemented to solve large scale problems. This method can be applied to solve real cases problem in order to assess the applicability of waste collection systems.

\section{References}

Alagöz, A. Z., \& Kocasoy, G. (2008). Improvement and modification of the routing system for the health-care waste collection and transportation in Istanbul. Waste Management, 28(8), 1461-1471.

Arnold, P., Peeters, D., Thomas, I., (2004). Modelling a rail/road intermodal transportation system. Transportation Research Part E: Logistics. 40(3), 255-270.

Ayed, H., Habbas, Z., Khadraoui, D., (2009, July). ACO for solving a multimodal transport problems using a transfer graph model. In IEEE Computers \& Industrial Engineering, 2009. CIE 2009. International Conference. 285-290.

Ayed, H., Galvez-Fernandez, C., Habbas, Z., Khadraoui, D., 2011. Solving time-dependent multimodal transport problems using a transfer graph model. Computers \& Industrial Engineering. 61(2), 391-401.

Bautista, J., Fernández, E., Pereira, J., (2008). Solving an urban waste collection problem using ant heuristics. Computers \& Operations Research. 35(9), 3020-3033.

Benjamin, A.M., and Beasley, J.E. (2010). Metaheuristics for the waste collection vehicle routing problem with time windows, driver rest period and multiple disposal facilities, Computers \& Operations Research, 37(12), 2270-2280.

Bing, X., de Keizer, M., Bloemhof-Ruwaard, J. M., \& van der Vorst, J. G. (2014). Vehicle routing for the ecoefficient collection of household plastic waste. Waste Management, 34(4), 719-729.

Bing, X., Bloemhof-Ruwaard, J. M., \& van der Vorst, J. G. (2014). Sustainable reverse logistics network design for household plastic waste. Flexible Service Manufacturing Journal, 26(1-2), 119-142.

Bodin, L. D., Golden, B. L., Assad, A. A., \& Ball, M. O. (1982). Special issue: Routing and scheduling of vehicles and crews. Comput. Oper. Res., 10(2), 62-211.

Chen, X., and Niu, H. (2011). Modeling Vehicle Routing Problem with Pick-Up Time Constraint for Multimodal Transportation, Proceedings of ICTE 2011, 464-469, Chengdu, China.

Dantzig, G. B., Ramser, J. H., (1959). The truck dispatching problem. Management Science, 6(1), 80-91.

Faccio, M., Persona, A., Zanin, G., (2011). Waste collection multi objective model with real time traceability data. Waste Management, 31(12), 2391-2405.

Galvez-Fernandez, C., Khadraoui, D., Ayed, H., Habbas, Z., Alba, E., (2009). Distributed approach for solving time-dependent problems in multimodal transport networks. Advanced Operations Research.

Ghose, M. K., Dikshit, A. K., \& Sharma, S. K. (2006). A GIS based transportation model for solid waste disposal-A case study on Asansol municipality. Waste Management, 26(11), 1287-1293.

Hemmelmayr, V. C., Doerner, K. F., Hartl, R. F., \& Vigo, D. (2013). Models and Algorithms for the Integrated Planning of Bin Allocation and Vehicle Routing in Solid Waste Management. Transportation Science, 48(1), 103-120.

Holland, John H., (1975). Adaptation in natural and artificial systems: An introductory analysis with applications to biology, control, and artificial intelligence. University of Michigan Press.

Hsieh, Y. C., \& You, P. S. (2014). An Artificial Intelligence Approach for the Solid Waste Collection Problem. Applied Mathematics, 8(1L), 283-291.

Kuo, R.J., Zulvia, F. E., and Suryadi, K. (2012). Hybrid particle swarm optimization with genetic algorithm for solving capacitated vehicle routing problem with fuzzy demand-A case study on garbage collection system, Applied Mathematics \& Computation, 215(5), 2574-2588.

Liu, J., He, Y., 2012, November. (2012). Ant colony algorithm for waste collection vehicle arc routing problem with turn constraints. In $8^{\text {th }}$ Int. Conf. Computational Intelligence and Security, IEEE. 35-39.

Liu, C.Y. (2013). An Improved Adaptive Genetic Algorithm for the Multi-depot Vehicle Routing Problem with Time Window. Journal of Network, 8(5), 1035-1042.

Lowe, D., (2005). Intermodal freight transport. Oxford (United Kingdom): Elsevier, ISBN 0750659351. 
Maimoun, M. A., Reinhart, D. R., Gammoh, F. T., McCauley Bush, P., (2013). Emissions from US waste collection vehicles. Waste management, 33(5), 1079-1089.

Moustafa, A., Abdelhalim, A. A., Eltawil, A. B., Fors, N., (2013), January. Waste Collection Vehicle Routing Problem: Case Study in Alexandria, Egypt. In The 19th International Conference on Industrial Engineering and Engineering Management, Springer Berlin Heidelberg, 935-944.

Nema, A. K., \& Gupta, S. K. (1999). Optimization of regional hazardous waste management systems: an improved formulation. Waste Management, 19(7), 441-451.

Nuortio, T., Kytöjoki, J., Niska, H., Bräysy, O., (2006). Improved route planning and scheduling of waste collection and transport. Expert Syst. Appl. 30(2), 223-232.

Ramos, T. R. P., Gomes, M. I., \& Barbosa-Póvoa, A. P. (2014). Economic and environmental concerns in planning recyclable waste collection systems. Transportation Research Part E: Logistics, 62, 34-54.

Sahoo, S., Kim, S., Kim, B. I., Kraas, B., \& Popov, A., (2005). Routing optimization for waste management. Interfaces, 35(1), 24-36.

Saleh, Y., Tofigh, A., \& Zahra, A. (2014). Transportation Routing in Urban Environments Using Updated Traffic Information Provided through Vehicular Communications. Journal of Transportation Systems Engineering and Information Technology, 14(5), 23-36.

Salhi, S., Imran, A., Wassan, N. A., (2013). The Multi-Depot Vehicle Routing Problem with Heterogeneous Vehicle Fleet: Formulation and A Variable Neighborhood Search Implementation. Computers \& Operations Research, In Press.

Siddiqui, A. W., \& Verma, M. (2015). A bi-objective approach to routing and scheduling maritime transportation of crude oil. Transportation Research Part D: Transport and Environment, 37, 65-78.

Sniezek, J., Bodin, L., (2006). Using mixed integer programming for solving the capacitated arc routing problem with vehicle/site dependencies with an application to the routing of residential sanitation collection vehicles. Annals of Operations Research, 144(1), 33-58.

Tavares, G., Zsigraiova, Z., Semiao, V., \& Carvalho, M. D. G. (2009). Optimisation of MSW collection routes for minimum fuel consumption using 3D GIS modelling. Waste Management, 29(3), 1176-1185.

Tung, D. V., Pinnoi, A., (2000). Vehicle routing-scheduling for waste collection in Hanoi. European Journal of Operations Research, 125(3), 449-468.

Xiong, G., Wang, Y., (2012). Best routes selection in multimodal networks using multi-objective genetic algorithm. Journal of Combinatorial Optimization, 1-19.

Ziliaskopoulos, A., Wardell, W., (2000). An intermodal optimum path algorithm for multimodal networks with dynamic arc travel times and switching delays. European Journal of Operations Research, 125(3), 486-502. 